\title{
Experimental Study on the Effect of Using Smartphones on Pedestrian Flow in Straight Corridors
}

\author{
Xudong Li · Weiguo Song · Jun Zhang \\ State Key Laboratory of Fire Science, University of Science and Technology of China, Hefei, \\ China, \\ E-mail: lixd2019@mail.ustc.edu.cn,wgsong@ustc.edu.cn, junz@ustc.edu.cn
}

Received: 13 August 2021 / Last revision received: 5 Oktober 2021 / Accepted: 2 November 2021 DOI: $10.17815 / \mathrm{CD} .2021 .120$

\begin{abstract}
With the development of science and technology, smartphones are widely used in people's daily lives. An interesting phenomenon is that many pedestrians use smartphones while walking in the public places, which not only harm and even kill in some cases, but also affect the pedestrian traffic safety. At present, most studies focus on the pedestrians in the normal state that they don't use phones while walking. Few research has been done on the pedestrian flow when they use phones. Therefore, the experiment that the pedestrians use phones while walking in straight corridor was conducted to study the movement characteristics and compared with the normal one. From the trajectories, the lane formation can be found in all experiments and the trajectories when they use phones are more chaotic. When pedestrians distract themselves by using phones, they walk more slowly and the flow is lower, leading to the longer egress time to pass the corridor. The distance from the boundary is defined as the shortest distance between the pedestrians and the wall. When they use phones, they try to avoid collision with the wall and walk away from the wall, so the distance is further than the normal one. The nearest pedestrian distance is defined as the nearest distance among all pedestrians. When they use phones, they distract themselves and don't have enough time to avoid collision with others, so the nearest pedestrians distance is closer than the normal one. Our findings maybe a new insight for pedestrian flow when they distract themselves by using the phones, talking with others and thinking deeply, which can enrich empirical data and contribute to the simulation model.
\end{abstract}

Keywords Smartphone $\cdot$ straight corridors $\cdot$ lane $\cdot$ egress time $\cdot$ border distance $\cdot$ nearest pedestrian distance 


\section{Introduction}

With the development of science and technology, smartphones become more and more popular. According to the report released by research firm Strategy Analytics (SA), as of June 2021, there will be 4 billion people around the world who own smartphones [1], which has been a new addiction upon adolescents [2]. The smartphones bring convenience to people's daily life, but cause lots of traffic safety problems while pedestrians use smartphones on the road. Cities like Washington D.C. and Chongqing, have set up the "only for smartphones user sidewalk" to prevent the accidents [3] . The pedestrians using smartphones on the road have been attracted wide attention and become a hot topic in traffic safety.

In pedestrian evacuation dynamic, some researches focus on the safety of pedestrian traffic to study the pedestrian movement characteristics, including the fundamental diagram [4-8], lane formation [9-11] and so on. In these studies, the distracted behaviors [12] are ignored. Some studies have considered the effects of mobile phone use on pedestrian traffic safety. Nasar et.al [13] analyzed the data from the US Consumer Product Safety Commission on injuries in hospital emergency rooms from 2004 through 2010. They found that the mobile-phone related injuries among pedestrians increased relative to total pedestrian injuries and using a mobile phone while walking puts pedestrians at risk of accident, injury or death. Pesic et.al [14] conducted field observation to find out how the use of mobile phone (talking, texting and listening to music) affects the behavior of pedestrians while they are crossing the street. The result showed that the pedestrians who use mobile phones while crossing the street behave less safely than the pedestrians who do not use mobile phones and that their safety depends on the way they use mobile phones. Mobile phone talking has the greatest effect on the unsafe behavior of pedestrians; texting/ viewing content on mobile phone also influences the pedestrians' behavior though less than speaking, while listening to music has the smallest impact. Hatfield et.al [15] conducted an observational field survey to compare the safety of crossing behaviors for pedestrians using, versus not using, a mobile phone. The results show that talking on a mobile phone is associated with cognitive distraction that may undermine pedestrian safety. Melissa et.al [16] used the multi-agent models that relate reported changes to the locomotion patterns and sensory abilities of distracted pedestrians to the corresponding parameters of a commonly used crowd simulation steering approach. They find even a few of these behaviors significantly alters the flow patterns of the simulated agents.

Besides, virtual reality is widely used to study the impact of smartphone distraction on pedestrians' movement. Schwebel et.al [17] considered the impact of distraction while talking, text-messaging, or listening in an interactive, semi-immersive virtual pedestrian street. They found that the pedestrians distracted by music or texting were more likely to be hit by a vehicle and the multimedia devices has a small but meaningful impact on college students' pedestrian safety. Sobhani et.al [18] considered three different conditions:1) not distracted , 2) distracted with a smartphone, and 3) distracted with a smartphone with a virtually implemented safety measure on the road. They find the females have more dangerous crossing behavior especially in distracted condition and the smart LED light safety treatment indeed improves the safety of distracted pedestrians. Joan et.al 
[19] studied the children's crossing intersections in VR program considering distractions (e.g., noise, pedestrians, park) and discussed possibilities for future VR interventions for injury prevention.

At present, most controlled experiments focus on the movement of pedestrian in normal walking condition, only a few consider the effect of distracted behaviors in field observation and VR environment. Also the empirical data applied to the simulation model are lacking. Hence, it is necessary to investigate the distracted behaviors in controlled experimental conditions, which can support the empirical data of pedestrian flow, as well as model development and validation. What's more, the straight corridor is a common geometric structure and widely used for collecting pedestrian movement data [5,20-23].

Based on these considerations, the experiment that the pedestrians use phones while walking in straight corridor was conducted to study the movement characteristics and compared with the normal one. In Sec. 2, the experiment setup is briefly described. In Sec. 3, the movement characteristics are analyzed and show results. Finally, Sec. 4 summarizes the paper and makes a conclusion.

\section{Set of the experiment}

The controlled experiments were conducted in September 2019 at the university of Science and Technology of China in Hefei, China. A total of 94 adults (undergraduate and graduate students) were recruited to participate in the experiment. The ratio of male and female is about 1:1. The illustration and a screenshot of the experiment are shown in Fig. 1. The movement of the adults in straight corridor under controlled conditions were studied. The length and the width of the channel is $10 \mathrm{~m}$ and $1.8 \mathrm{~m}$ respectively, which was built by using partitions with a height of $1.8 \mathrm{~m}$ and width of $0.8 \mathrm{~m}$. The coordinate system is established from the starting point of the lower left corner of the channel as the origin. In order to investigate the effect of different measurement area on the results, three regions in different position were selected as shown in Fig. 1(a). The entrance of the corridor $(x \in[0,2 \mathrm{~m}])$ is region $a$, the middle $(x \in[4 \mathrm{~m}, 6 \mathrm{~m}])$ is region $b$ and the exit $(x \in[4 \mathrm{~m}, 6 \mathrm{~m}])$ is region $c$. During the experiment, the volunteers were asked to wear a red or orange hat for extracting the trajectories precisely by software Petrack [16].

The experiments were mainly conducted in two types of scenes. One is the normal walking experiment. The volunteers were asked to stand in line in the waiting area before starting the experiment. Then the starter gave a command to start the experiment. During this experiment, the volunteers were asked to walk as usual through the straight corridor in normal state, in this case, some behaviors such as talking to others, using smartphones and pushed others are not allowed. The other is using smartphones while walking. The volunteers were asked to stand in line in the waiting area before starting the experiment. They were required to distract themselves by using smartphones. The specific ways of using smartphones were not restricted according to their personal preferences like games, chatting and so on. When the starting command was given, they kept using the smartphones though the straight corridor.

The whole process of the experiments was recorded by two digital cameras with the res- 
olution of $1920 * 1080$ and the frame rate of $25 \mathrm{fps}$.

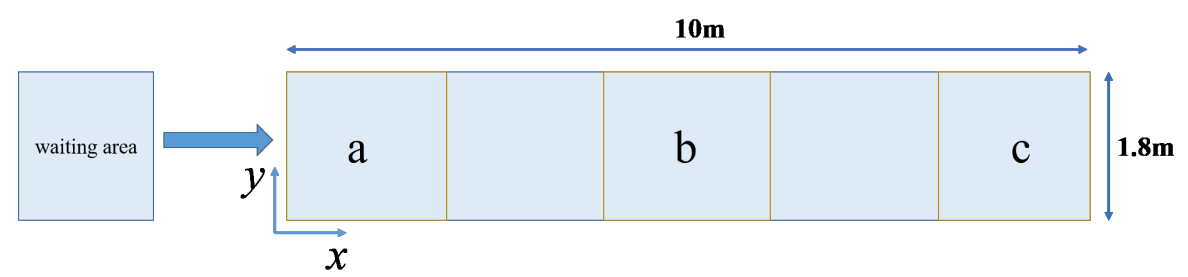

(a)

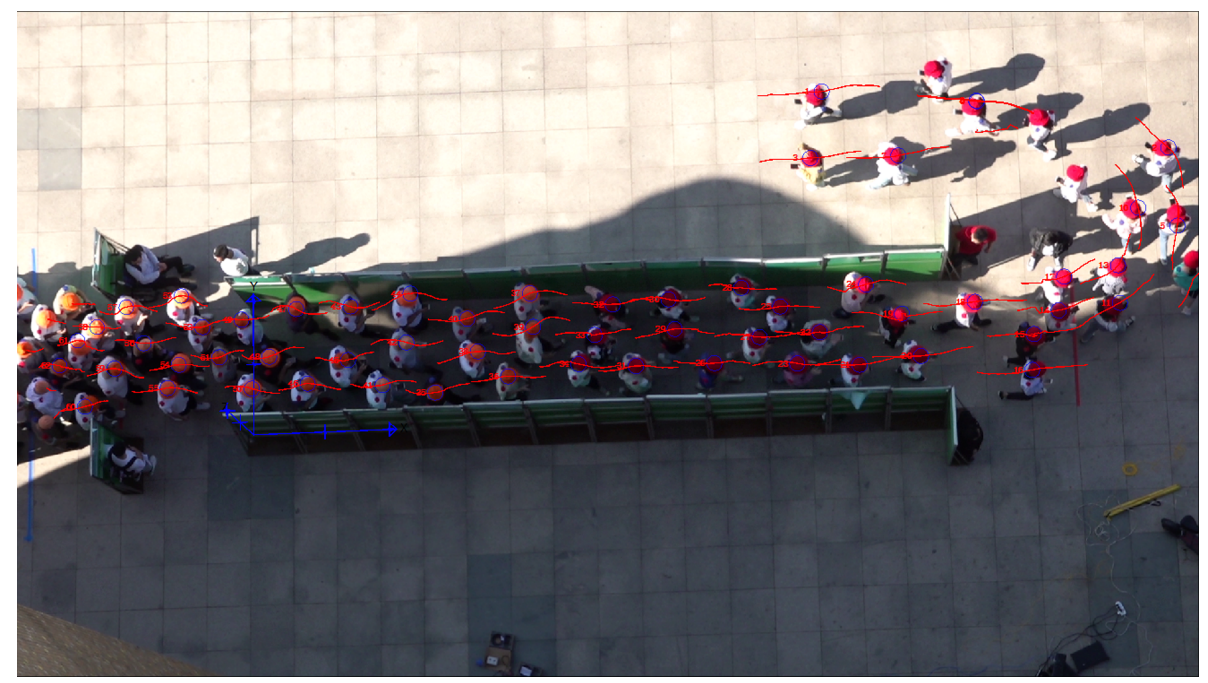

(b)

Figure 1 The sketch (a) and a screenshot (b) of the experiment.

\section{Analysis and results}

\subsection{Trajectories}

In the experiments, the pedestrians were asked to walk through the corridor and their trajectories were obtained. Based on the trajectories, the individual instantaneous velocity $\vec{v}_{i}(t)$ is calculated with Eq. 1.

$$
\vec{r}_{i}(t)=\frac{\vec{r}_{i}(t+m)-\vec{r}_{i}(t-m)}{2 m}
$$

Hereinto, $\vec{r}_{i}(t+m)=\left(x_{i}(t+m), y_{i}(t+m)\right)$ is the position of a pedestrian at the time of $t+m, m$ is a constant, and in this paper $m=0.2 s$ [17].

The pedestrians' trajectories obtained from the video recordings with instantaneous speed in different scenarios are shown in Fig. 2. Different color represents the speed of the pedestrian at a certain position. It shows that the three lanes are obvious when they walk in the same direction, which means three pedestrians are allowed to walk side by 
side in the corridor. When the pedestrians walk as normal, the lane formation is more obvious and the trajectories cross is not chaotic. From the color of the trajectories, the speed of normal walking is higher than using smartphones while walking.

To study the lane formation in the corridor, the probability distribution diagrams of pedestrians in y-direction are shown in Fig. 3. It can be seen there are three obvious peaks of probability distribution diagram corresponding to three lanes when they walk as normal. The peaks of two sides are higher than that in the middle. The position of three lanes is $0.3 \mathrm{~m}, 0.9 \mathrm{~m}$ and $1.4 \mathrm{~m}$, respectively. Three peaks of probability distribution diagram are obvious and the peaks are relatively close while walking though the corridor. The pedestrians on both sides of the corridor moved towards the middle, which increases the probability of pedestrians close to middle lane. The position of three lanes is $0.3 \mathrm{~m}, 0.8 \mathrm{~m}$ and $1.4 \mathrm{~m}$, respectively. Therefore, the lane formation will be appeared in two scenarios and the position of lane is close. When the pedestrians used smartphones while walking, they looked down as the phone and might ignore the walking route. The pedestrians on both sides of the corridor might rely on their own perception to make the actual trajectories deviate to a certain extent, resulting in the intersection of trajectories between the middle lane and the lanes on both sides. The distinction among lanes when they walk as normal is more obvious than using phones while walking.

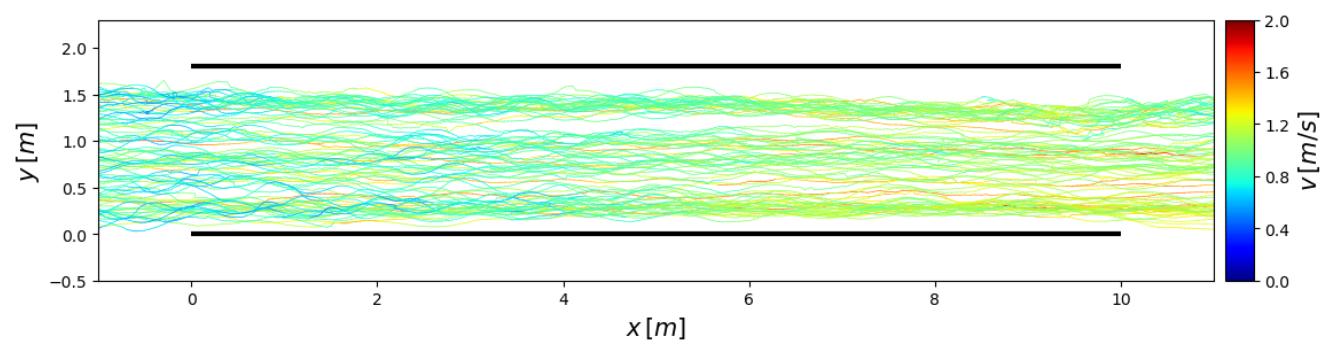

(a) normal

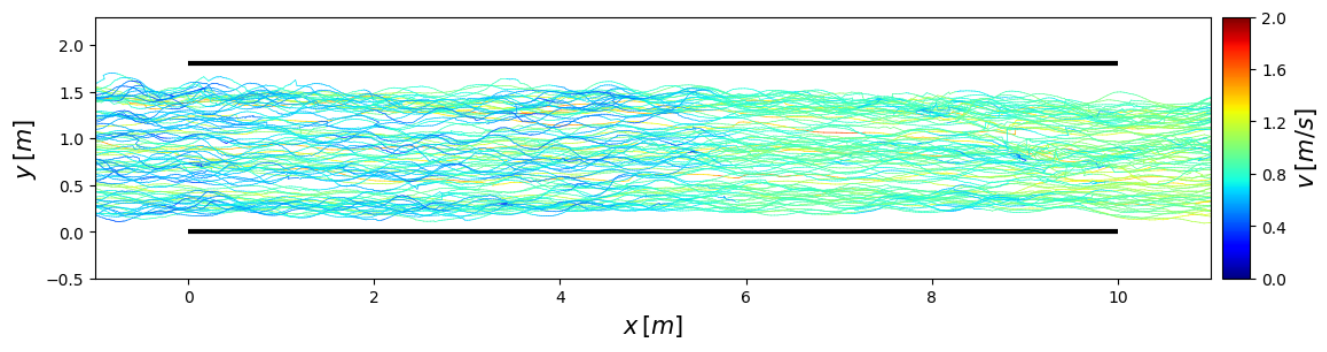

(b) phone

Figure 2 Trajectory with instantaneous velocity in different scenarios. (a) The pedestrians were walking at the normal state. (b)The pedestrians were using smartphones while walking though the corridor.

To further study the influence of using smartphones on pedestrians' trajectories, the trajectory offset $\Delta y$ is defined as the absolute value of y coordinate difference when the pedestrians entered the corridor $(x=0)$ and exited the corridor $(x=10)$. It can be calculated by $\Delta y=\left|y_{x=0}-y_{x=10}\right|$. The trajectory offset probability distribution is shown in 
Fig. 4. It shows that more than half of the pedestrians can keep walking in a straight line and the trajectory offset is within $0.1 \mathrm{~m}$ when they walk as normal. As the trajectory offset is more than $0.1 \mathrm{~m}$ and keeps increasing, the proportion of pedestrians using phone while walking is larger than the normal. The trajectory offset is within $0.4 \mathrm{~m}$ when they walk as normal, while the trajectory offset of some pedestrians using phones is more than $0.4 \mathrm{~m}$.

Therefore, the pedestrians using smartphones have larger trajectory offset. If they do not pay attention to the surrounding environment during walking, they are likely to collide with pedestrians and objects around them.

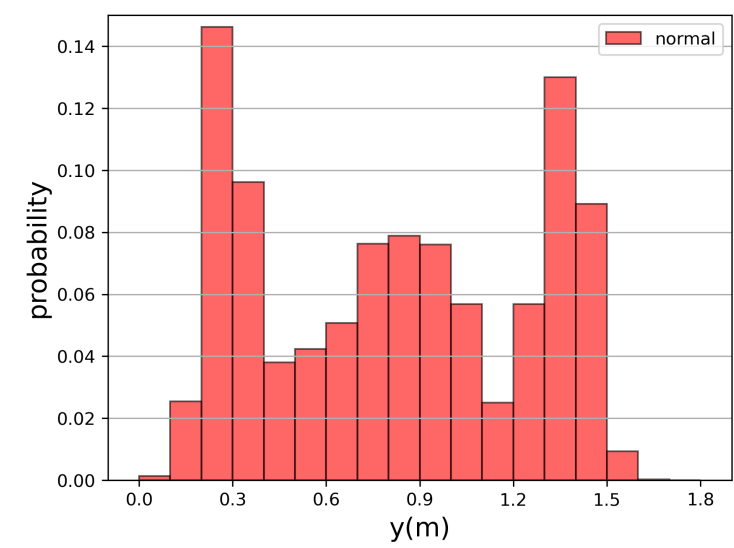

(a) normal

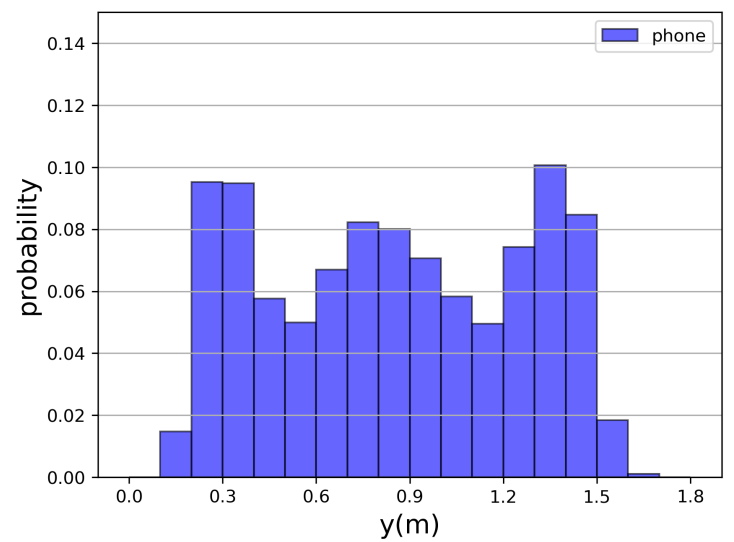

(b) phone

Figure 3 The probability distribution diagram of pedestrians in $y$-direction. (a) The pedestrians were walking at the normal state. (b)The pedestrians were using smartphones while walking though the corridor.

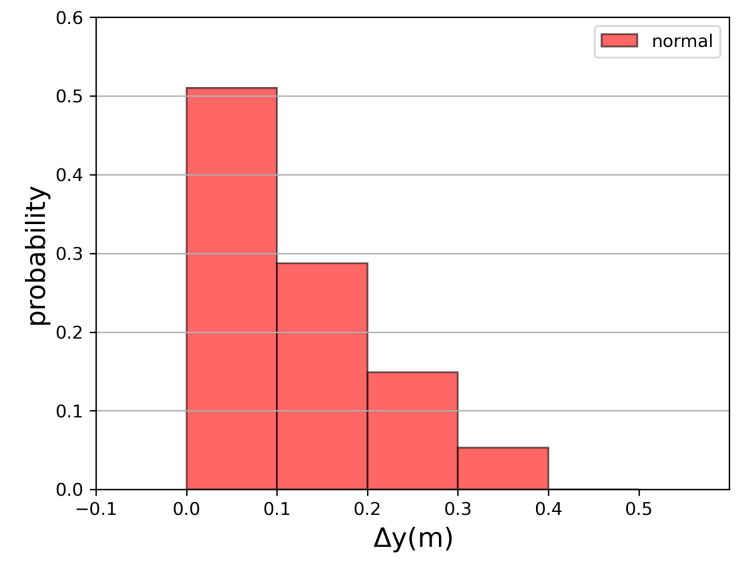

(a) normal

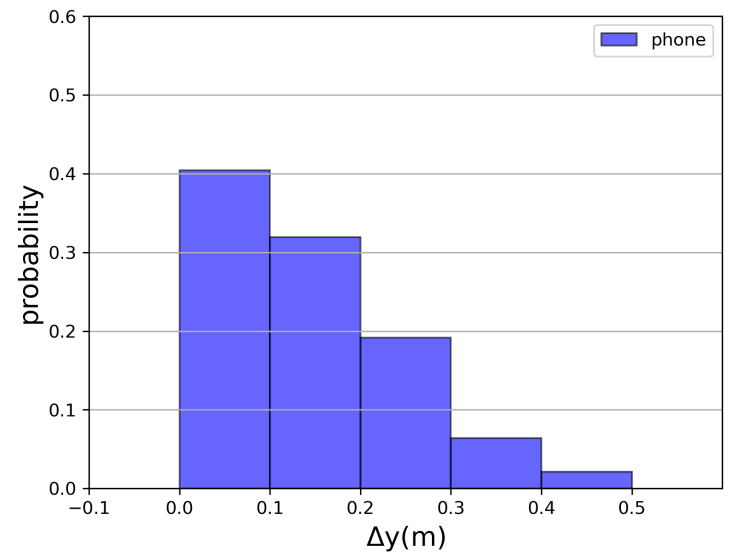

(b) phone

Figure 4 The trajectory offset probability distribution. 


\subsection{Velocity-density diagram}

In this study, three areas were selected as the measurement area $a, b$ and $c$. The length of the measurement area is $2 \mathrm{~m}$ and the width of the measurement area is $1.8 \mathrm{~m}$. The velocity and density in different measurement areas is calculated by using Voronoi method [4].

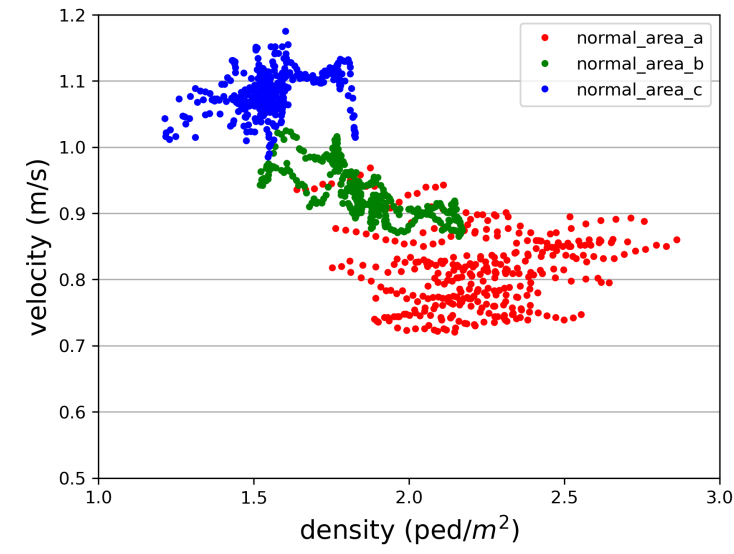

(a) normal

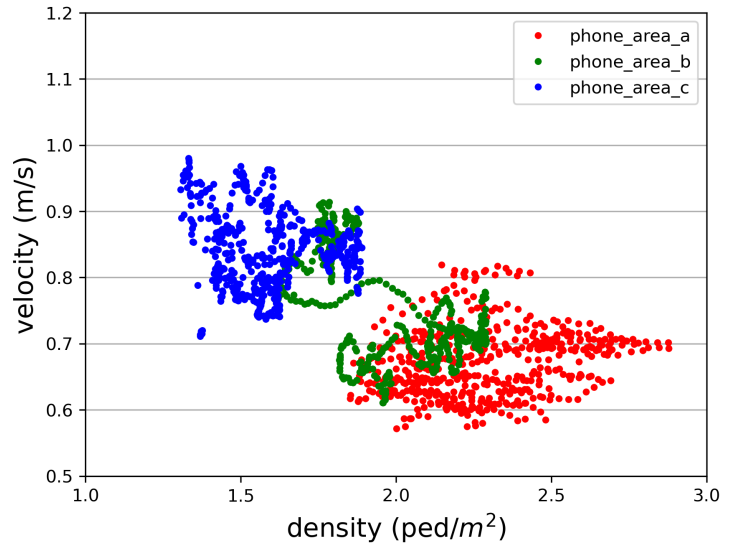

(b) phone

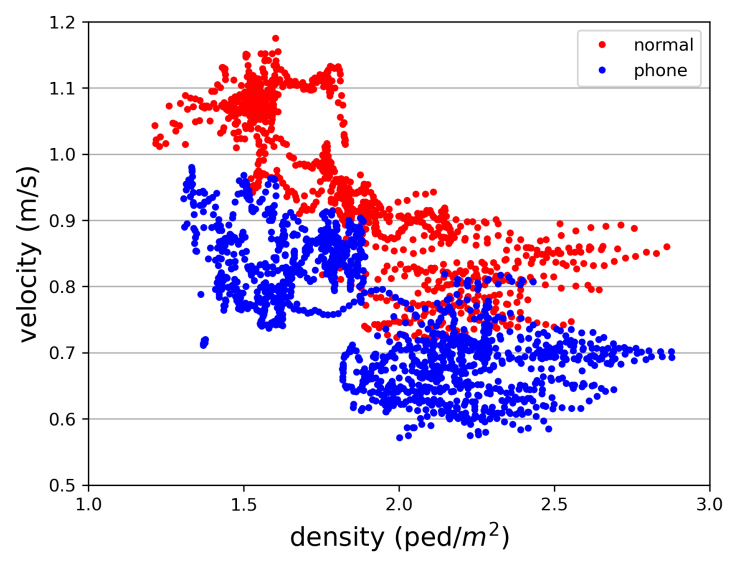

(c) normal and phone

Figure 5 The velocity and density in different areas. (a) The pedestrians were walking at normal state. (b) The pedestrians were using smartphones while walking though the corridor. (c) This figure shows (a) and (b).

The velocity and density in stable stage are shown in Fig. 5. It shows when walking in a normal way and using phone, the velocity in area $a$ is larger than that in area $b$ and the velocity in area $b$ is larger than that in area $c$, which is similar to the density in stable stage. In area $a$, the pedestrians started walking at the initial stage. When entering this measurement area, the pedestrians will accelerate until they reach the steady speed. The pedestrians stayed in this area for a long time, resulting in a relatively high density. In area $c$, the pedestrians kept a certain walking speed from entering the measurement area to leaving the measurement area. The pedestrians can pass though the measurement area 
at a faster speed, resulting in a relatively small density. The result in area $b$ is between $b$ and $c$.

The velocity-density diagram under different walking styles is shown in Fig. 5(c). The density varies from about form $1.2 \mathrm{ped} / \mathrm{m}^{2}$ to $2.8 \mathrm{ped} / \mathrm{m}^{2}$. The speed decreases as the density increases, which due to the distance among pedestrians decreases as the density increases. When the pedestrians made the decision, they were influenced by the distance to others. The larger distance is more benefit for walking as usual. When the density is relatively small, the speed in normal state is obvious higher than the speed in using phone while walking. When the speed is large, a small part of normal walking speed close to the speed in using phones.

To compare the speed in each measurement area, the boxplot of the speed is shown in Fig. 6. It seems the speed increases as the position $x$ increases. To investigate the numerical relation between the speed and the position $x$, the linear regression is adopted. In the normal condition, the fitting relation can be expressed as $v=0.03 x+0.78$. When using the phones, the fitting relation can be expressed as $v=0.02 x+0.65$. To study the difference of speed in two conditions, the Mann-Whitney test is adopted. The result in area $a, b$ and $c$ are all $p<0.05$, which means the speed in using phones while walking shows significant difference with the normal state. In all areas, the speed in normal state is larger than using the phone while walking, which might due to the pedestrian's attention is distracted by smartphones.

On the whole, the speed in normal state is larger than using smartphones while walking, which is due to the pedestrian's attention is distracted by smartphones and influenced by the density, leading to lower speed. So the pedestrians' speed decreases as the density increases, and using smartphones while walking significantly reduces the pedestrians' speed. 


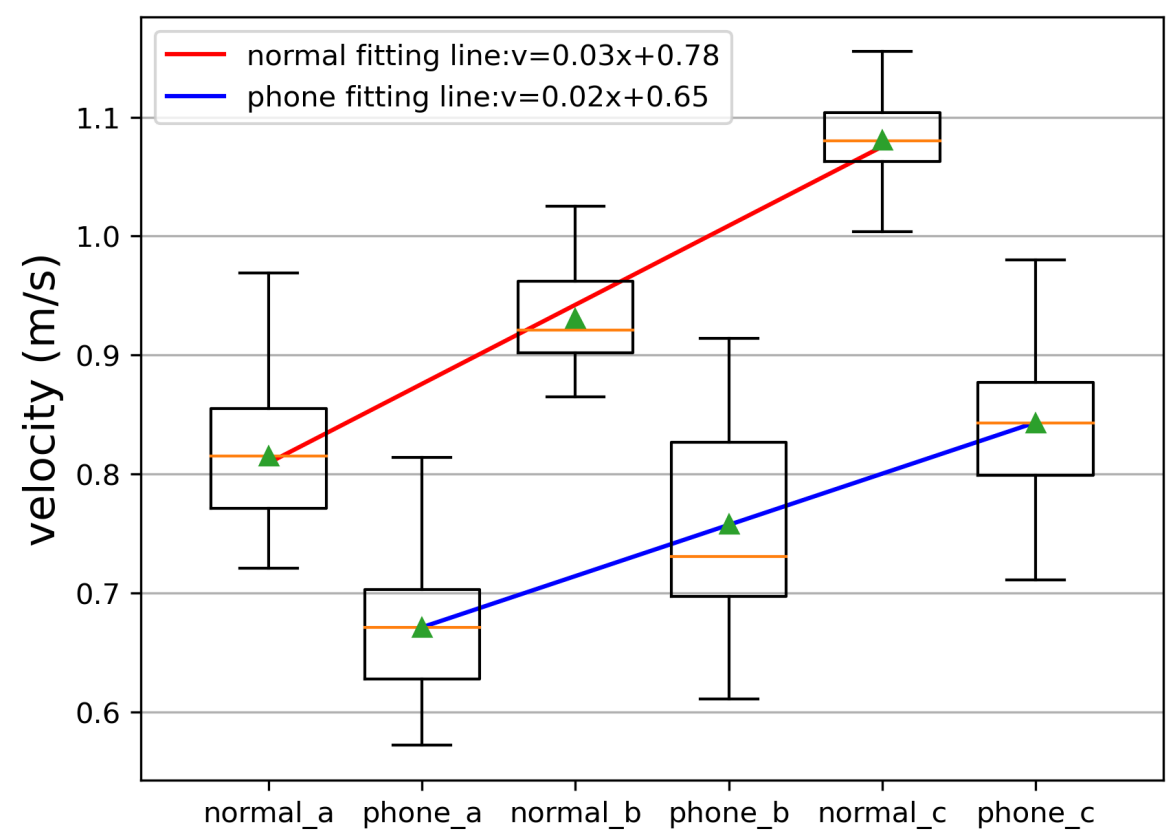

Figure 6 The boxplot of velocity.

\subsection{Flow}

To compare the capacity of the two scenes, the reference line is selected at the position of $x=5 \mathrm{~m}$. The number of pedestrian passing the reference line over time was shown in Fig. 7 . When the experiment started, the pedestrians walked from the starting position to the reference line, then passed though the reference line and gone out of the corridor. When the pedestrians started to pass though the reference line, the number of pedestrians increases over time until all pedestrians passed though the reference lines. When the pedestrians walked as normal, the whole process lasts from $6 \mathrm{~s}$ to $35.08 \mathrm{~s}$. When the pedestrians used phones while walking, the whole process lasts from $6.2 \mathrm{~s}$ to $41.92 \mathrm{~s}$. The little difference in the time of the first pedestrian passing the line is caused by the effect of using phones on pedestrians' speed. By calculating the average flow during the whole process, the average flow under normal condition is $3.23 \mathrm{ped} / \mathrm{s}$, while the average flow under using phone condition is $2.63 \mathrm{ped} / \mathrm{s}$.

Besides, the specific flow is used to evaluate the flow in an area rather than the reference line. To study the flow rate throughout the corridor, the specific flow is calculated by the Voronoi method [26] over small regions $(20 \mathrm{~cm} * 20 \mathrm{~cm})$. The result is shown in Fig. 8. From the heatmap, it shows that the color in some regions in normal state is darker, which mean that specific flow is larger than that in the using phones state. That is due to the speed in normal state is larger than that in the using phones states.

Therefore, the average flow under normal condition is larger than using the phone while walking. Using smartphones will reduce the average flow and cause influence on the movement of the whole crowd. 


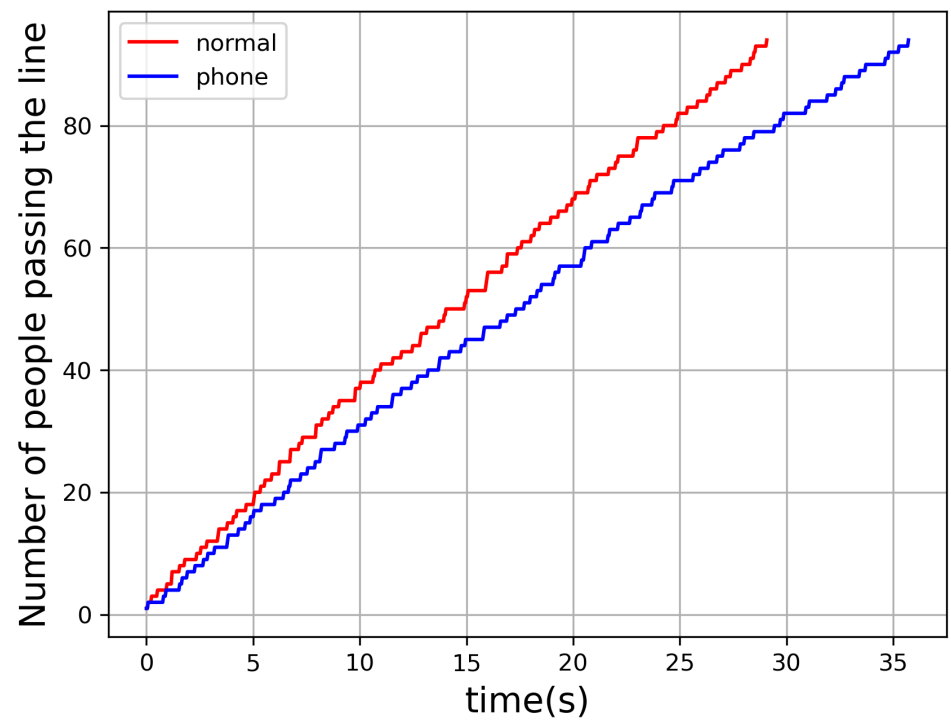

Figure 7 Number of pedestrians pass though the reference line.

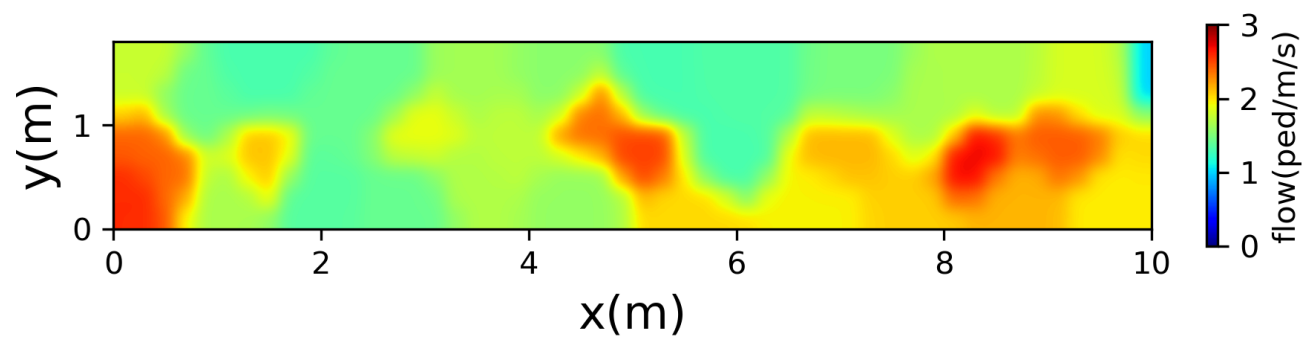

(a) normal

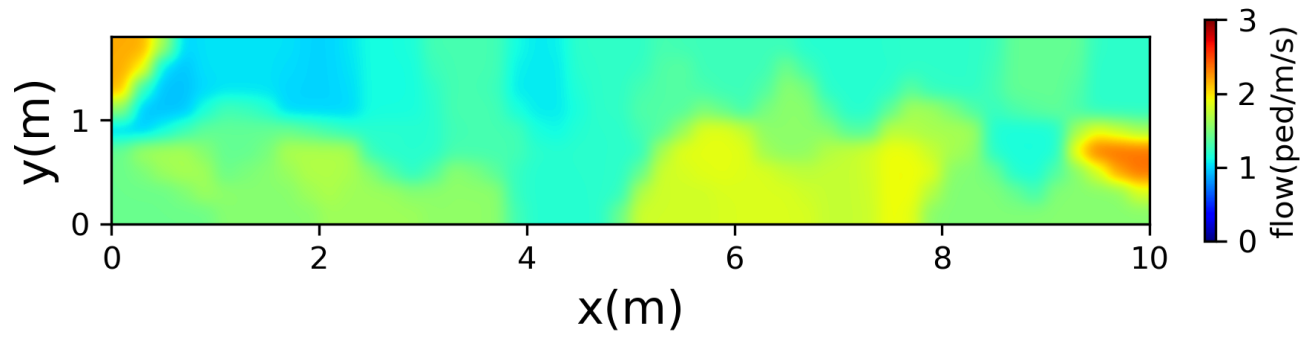

(b) phone

Figure 8 The heatmap of the flow rate throughout the corridor.

\subsection{Distance from the boundary}

Generally, the pedestrians would keep a certain distance from the boundary. In the previous section, the densities are close in the three measurement areas. The distance from 
the boundary is defined as the minimum distance between the pedestrian's head and the boundary on two sides in different measurement areas.

In the density stable stage, the distance from the boundary is shown in Fig. 9. To study the difference of distance in two conditions, the statistical test is adopted. The result in area $a, b$ and $c$ are all $p<0.05$, which means the distance in using phones while walking shows significant difference with the normal state. In different areas, the average distance from the boundary is larger than that using smartphones while walking. On the one hand, the trajectory will be offset and the offset is larger than the normal condition. From the previous lane formation analysis, the pedestrians on both sides tended to move to the middle, resulting in the increase of the distance from the boundary. On the other hand, the pedestrians who using smartphones while walking would try to avoid collision with the boundary psychologically and they might be familiar with the scene to a certain extent at the start stage, which made them move to the middle of the corridor. The pedestrians walking as normal could try to keep in a straight line, as long as the distance from the boundary is within a certain range. They would not change their walking route deliberately to avoid the conflict with the boundary.

Therefore, the pedestrians using smartphones while walking in straight corridor are more likely to move to the middle to avoid colliding with the boundary.

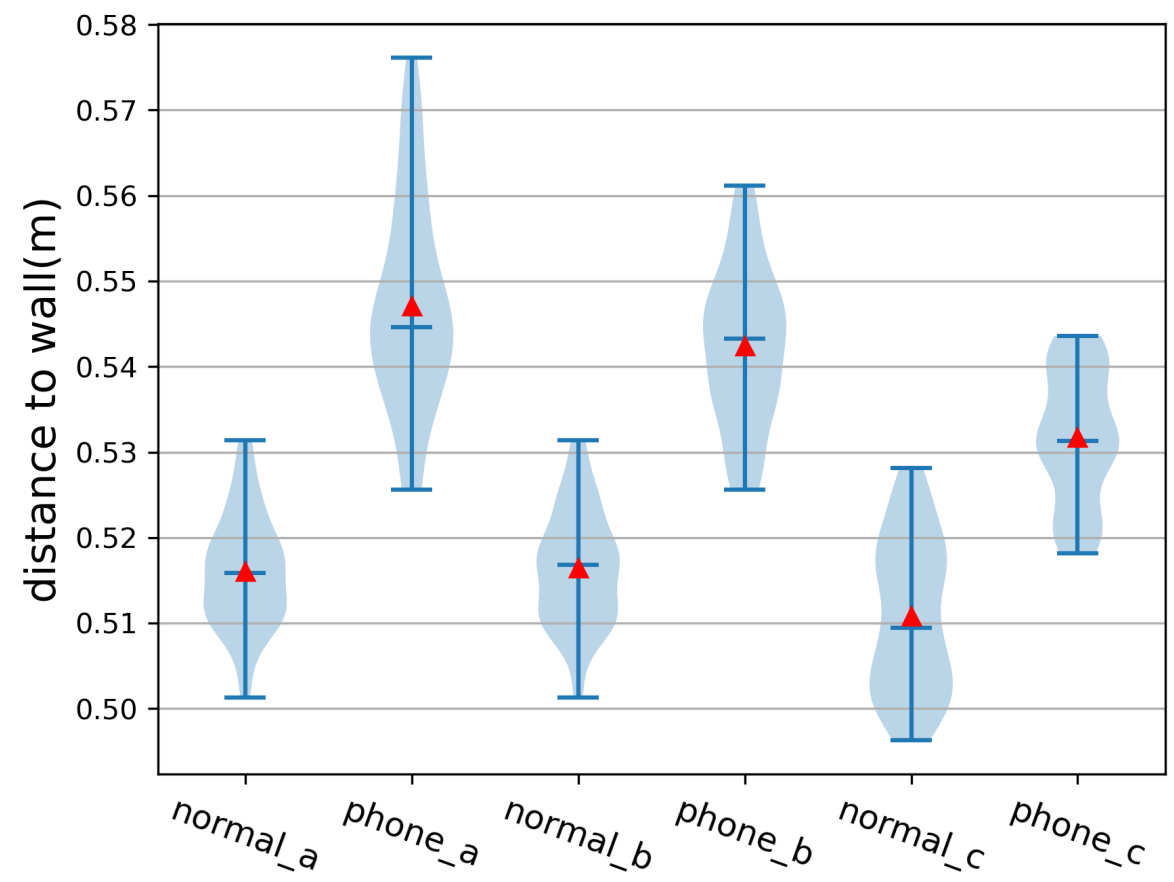

Figure 9 The distance between the pedestrians and the boundary in different areas.

\subsection{Nearest distance among pedestrians}

The pedestrians prefer to keep a certain distance from surrounding objects to avoid collisions, which makes them feel comfortable. In the previous section, the densities are close 
in three measurement areas. The nearest distance among pedestrians is defined as the minimum distance between the chosen pedestrian and other pedestrians, which is calculated by the head trajectory.

In the density stable stage, the nearest distance among pedestrians is shown in Fig. 10. To study the difference of nearest distance in two conditions, the statistical test is adopted. The result in area $a, b$ and $c$ are all $p<0.05$, which means the nearest distance in using phones while walking shows significant difference with the normal state. In different areas, the mean of the nearest distance among using smartphones pedestrians is less than the normal condition. Although the pedestrians will keep a certain distance from others, they were distracted by using smartphones and react slowly to the movement of pedestrians around them to some extent. As a result, the pedestrians keep walking and shorten the distance to others.

Therefore, using smartphones while walking will reduce the nearest distance among pedestrians, which might increase the probability of collision and conflict with others.

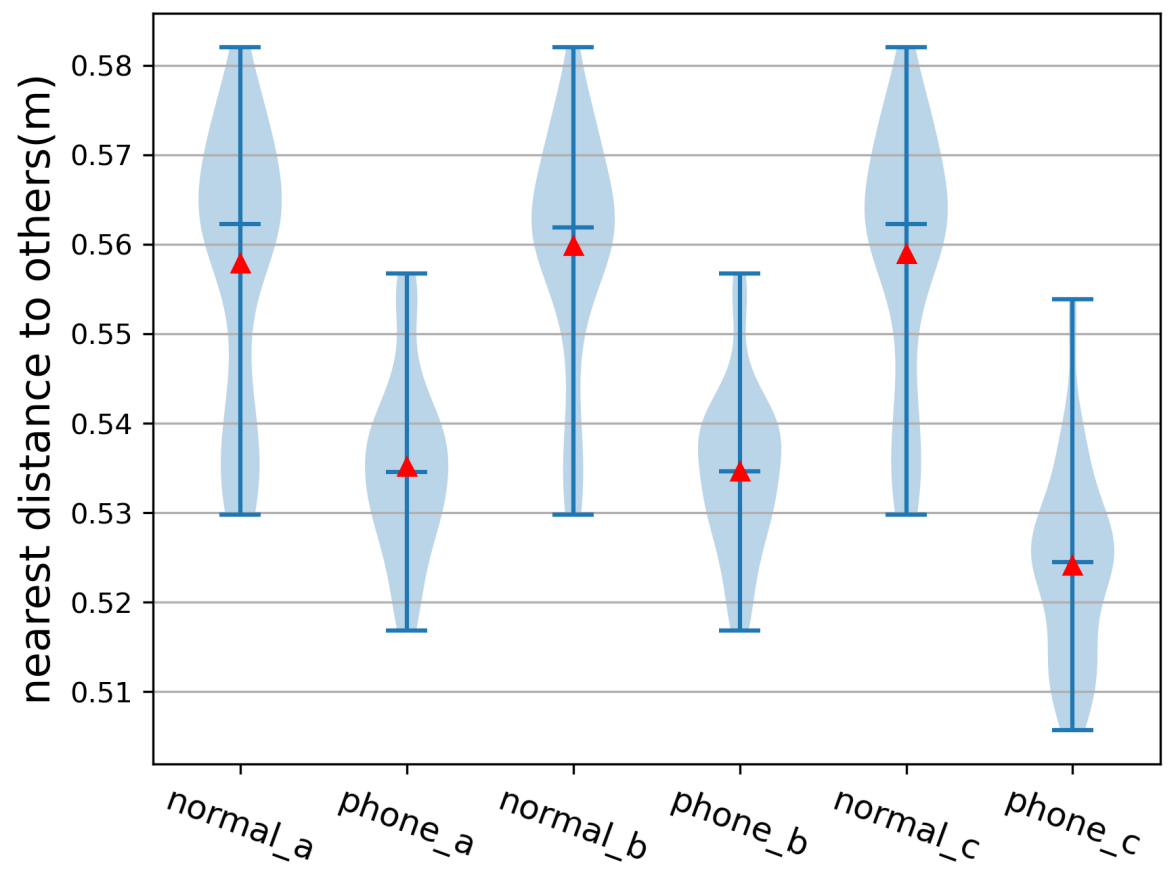

Figure 10 The nearest distance among pedestrians in different areas.

\section{Summary}

In this paper, a series of experiments were carried out to investigate the effect of using smartphones on uni-directional pedestrian flow in straight corridor. The movement characteristics are analyzed and compared with the normal one. Based on the trajectory data, the lane formation can be found in all experiments and the trajectory when they use phones is more chaotic. The pedestrians using mobile phones while walking have larger trajectory offset. 
When pedestrians distract themselves by using phones, they walk more slowly and the flow is smaller, leading to the longer egress time to pass the corridor. The boundary distance is defined as the shortest distance between the pedestrians and the wall. When they use phones, they would try to move to the middle of the corridor and avoid collision with the wall, so the boundary distance is further than the normal one.

The nearest pedestrian distance is defined as the nearest distance among all pedestrians. When they use phones, they distract themselves and don't have enough time to avoid collision with others, so the nearest pedestrians distance is less than the normal one. Although they avoid the collision with the boundary, they are more likely to collide with other pedestrians. In short, when they use smartphones while walking, they are more likely to collision with the moving objects.

Our study has carried out a straight corridor experiment to study the effect of using smartphones on the movement characteristics of pedestrian traffic. The findings maybe a new insight for pedestrian flow when they distract themselves by using the phones, talking with others and thinking deeply, which can enrich empirical data and contribute to the simulation model.

In the future, more scenes including single-file, bottlenecks, uni-and bidirectional, Tjunction, Y-junction will be carried out to obtain richer movement characteristics of pedestrian traffic and more different ways of using phones will be considered, thus providing more basic theoretical basis for pedestrian traffic safety.

Acknowledgements This research was supported by the National Natural Science Foundation of China (52074252); the Key R\&D Program of Anhui Province(202004a07020052).

\section{References}

\section{References}

[1] Strategy analytics: Half the world owns a smartphone. Strategy Analytics: Half the World Owns a Smartphone - Strategy Analytics. (n.d.). Retrieved December 14, 2021, from https://news.strategyanalytics.com/press-releases/press-releasedetails/2021/Strategy-Analytics-Half-the-World-Owns-a-Smartphone/default.aspx

[2] S. Halayem,O.Nouira, S. Bourgou, A. Bouden, S. Othman, M. Halayem:The mobile: a new addiction upon adolescents. La Tunisie Médicale 88, 593-596 (2010)

[3] lutian. Afraid of "phubbers"! Chongqing is now the first mobile phone sidewalk in China. 2014; Available from: http://www.techweb.com.cn/world/2014-0915/2075855.shtml

[4] Jin C J, Jiang R, Wei W, et al:Microscopic events under high-density condition in uni-directional pedestrian flow experiment. Physica A: Statistical Mechanics and its Applications 506, 237-247 (2018), doi:10.1016/j.physa.2018.04.030 
[5] Zhang J, Klingsch W, Schadschneider A, et al:Transitions in pedestrian fundamental diagrams of straight corridors and T-junctions. Journal of Statistical Mechanics: Theory and Experiment 06, P06004 (2011), doi:10.1088/1742-5468/2011/06/P06004

[6] Helbing D, Johansson A, Al-Abideen H Z:Dynamics of crowd disasters: An empirical study. Physical review E 75, 4 (2007), doi:10.1103/PhysRevE.75.046109

[7] Zhang,J., et al.: Ordering in bidirectional pedestrian flows and its influence on the fundamental diagram. Journal of Statistical Mechanics: Theory and Experiment, 02, P02002(2012), doi:10.1088/1742-5468/2012/02/P02002.

[8] Boltes, M., et al: Experimentation, data collection, modeling and simulation of pedestrian dynamics. In: Statistics, Probability \& Numerical Analysis 2014 - Methods \& Applications, 49-60(2014)

[9] Lian L, Song W, Richard Y K K, et al:Experimental study of high-density pedestrian flow field characteristics at a crossing. In:Traffic and Granular Flow'15,57-64(2016), doi:10.1007/978-3-319-33482-0_8

[10] Helbing D, Buzna L, Johansson A, et al:Self-organized pedestrian crowd dynamics: Experiments, simulations, and design solutions.Transportation science 39, 1 (2005), doi:10.1287/trsc.1040.0108

[11] Feliciani C, Nishinari $\mathrm{K}$ :Empirical analysis of the lane formation process in bidirectional pedestrian. flow.Physical Review E 94, 3 (2016), doi:10.1103/PhysRevE.94.032304

[12] Hedlund J, Simpson H M, Mayhew D R: International conference on distracted driving: Summary of proceedings and recommendations. In:CAA,2-5(2005)

[13] Nasar, J.L. and D. Troyer :Pedestrian injuries due to mobile phone use in public places. Accident Analysis \& Prevention 57, 91-95(2013), doi:10.1016/j.aap.2013.03.021

[14] Pešić, D., et al.:The effects of mobile phone use on pedestrian crossing behaviour at unsignalized intersections-Models for predicting unsafe pedestrians behaviour. Safety science 82,1-8(2016), doi:10.1016/j.ssci.2015.08.016

[15] Hatfield J, Murphy S :The effects of mobile phone use on pedestrian crossing behaviour at signalised and unsignalised intersections. Accident analysis \& prevention 39, 197-205 (2007), doi:10.1016/j.aap.2006.07.001

[16] Kremer, M., Haworth, B., Kapadia, M. et al :Modelling distracted agents in crowd simulations.The Visual Computer 37, 107-118 (2021), doi:10.1007/s00371-020-01969-4 
[17] Schwebel, D.C., et al.:Distraction and pedestrian safety: how talking on the phone, texting, and listening to music impact crossing the street.Accident Analysis \& Prevention 45, 266-271(2021), doi:10.1016/j.aap.2011.07.011

[18] Sobhani, A. and B. Farooq :Impact of smartphone distraction on pedestrians' crossing behaviour: An application of head-mounted immersive virtual reality.Transportation research part F: traffic psychology and behaviour 58, 228-241 (2018), doi:10.1016/j.trf.2018.06.020

[19] McComas, J., M. MacKay, and J. Pivik :Effectiveness of virtual reality for teaching pedestrian safety. CyberPsychology \& Behavior 5, 185-190 (2002), doi:10.1089/109493102760147150

[20] Ren X, Zhang J, Song W, et al :The fundamental diagrams of elderly pedestrian flow in straight corridors under different densities.Journal of Statistical Mechanics: Theory and Experiment 2,023403(2019), doi : 10.1088/1742-5468/a afa 7b

[21] Pan, H., et al :Fundamental diagram of pedestrian flow including wheelchair users in straight corridors.Journal of Statistical Mechanics: Theory and Experiment 3, 033411(2021), doi:10.1088/1742-5468/abe93f

[22] Lian, L., et al :Pedestrian merging behavior analysis: An experimental study. Fire Safety Journal 91,918-925(2017), doi:10.1016/j.firesaf.2017.04.015

[23] Ye, R., et al :Experimental study of pedestrian flow through right-angled corridor: uni-and bidirectional scenarios. Journal of Statistical Mechanics: Theory and Experiment 4, 043401(2019), doi:10.1088/1742-5468/aboc13

[24] Boltes, M., et al :Automatic extraction of pedestrian trajectories from video recordings. In Pedestrian and Evacuation Dynamics 2008, 43-54(2010) doi:10.1007/978-3-642-04504-2_3

[25] Lian, L., et al :An experimental study on four-directional intersecting pedestrian flows.Journal of Statistical Mechanics: Theory and Experiment 8, 08024(2019), doi:10.1088/1742-5468/2015/08/P08024

[26] Boltes M, Zhang J, Seyfried A, et al: T-junction: Experiments, trajectory collection, and analysis. In: IEEE International Conference on Computer Vision Workshops (ICCV Workshops), 158-165 (2011), 